\title{
Evaluation of Retinal Nerve Fiber Layer Thickness and Optic Nerve Head Parameters in Obstructive Sleep Apnoea Patients
}

\author{
Qi Zhe Ngoo, Nazihatul Fikriah A ${ }^{1}$, Baharudin $\mathrm{A}^{2}$, Wan Hazabbah WH \\ ${ }^{1}$ Department of Ophthalmology, School of Medical Sciences, Health Campus, Universiti Sains Malaysia, Kubang Kerian, Malaysia \\ ${ }^{2}$ Department of Otorhinolaryngology-Head and Neck Surgery, School of Medical Sciences, Universiti Sains Malaysia, Kubang \\ Kerian, Malaysia
}

Purpose: To study the retinal nerve fiber layer (RNFL) thickness and optic nerve head (ONH) parameters in obstructive sleep apnoea (OSA) patients and their relationship with severity of the disease.

Methods: A cross-sectional, hospital-based study. Fifty-four OSA subjects and 54 controls were recruited. Candidate that fulfil the criteria with normal ocular examinations then proceed with spectrum domain Cirrus optical coherence tomography examinations. ONH parameters and RNFL thickness were evaluated. Apnoea-hypopnoea index (AHI) of the OSA group were obtained from the medical record.

Results: In OSA, mean of average RNFL thickness was $93.87 \mu \mathrm{m}$, standard deviation (SD) $=9.17, p=0.008(p<0.05)$ while superior RNFL thickness was $113.59 \mu \mathrm{m}, \mathrm{SD}=16.29, p \leq 0.001(p<0.05)$. RNFL thickness fairly correlate with severity of the disease (AHI), superior RNFL with $\mathrm{R}=0.293, \mathrm{R}^{2}=0.087, p=0.030(p<0.05)$, and nasal $\mathrm{RNFL} \mathrm{R}=0.292, \mathrm{R}^{2}=0.085, p=0.032$. No significant difference and correlation observed on ONH parameters. In control group, mean of average RNFL thickness was $98.96 \mu \mathrm{m}, \mathrm{SD}=10.50, p=0.008(p<0.05)$ while superior RNFL thickness was $125.76 \mu \mathrm{m}, \mathrm{SD}=14.93, p \leq 0.001(p<0.05)$.

Conclusions: The mean of the average and superior RNFL thickness were significantly lower in the OSA group compare to control. Regression analysis showed RNFL thickness having significantly linear relationship with the AHI, specifically involving the superior and nasal quadrant.

Key Words: Apnea, Apnoea-hypopnoea index, Optic disk, Optic nerve ischemia, Retina

Obstructive sleep apnoea (OSA) syndrome is characterized by repetitive episodes of upper airway obstruction that occur during sleep, usually associated with a reduction in blood oxygen saturation [1]. The prevalence in Asian

Received: October 1, 2020 Final revision: April 20, 2021

Accepted: April 21, 2021

Corresponding Author: Wan Hazabbah WH, MD. Department of Ophthalmology, School of Medical Sciences, Health Campus, Universiti Sains Malaysia, Kubang Kerian, 16150 Kelantan, Malaysia. Tel: 60-12565-7953, E-mail: hazabbah@usm.my populations ranges from $3.57 \%$ to $15.5 \%$ [2-5].

Over the past two decades various studies have found the link between OSA and systemic diseases such as heart failure [6], diabetes mellitus [7], cognitive impairment [8], and ocular diseases; non arteritic anterior ischaemic optic neuropathy [9-11], glaucoma [12-15], retinal vein occlusion [16] and, central serous chorioretinopathy [17,18].

Chronic oxygen deprivation in OSA may results in activation of the sympathetic pathway, vascular endothelial dysfunction, increased oxidative stress, and inflammation, which may contribute to the initiation and progression of 
vascular diseases. It is postulated that there is a reduction of ocular perfusion pressure and decreased oxygenation to the optic nerve, causing ischaemia particularly to the sensitive ganglion cell layer resulting in optic neuropathy in OSA patients [19].

Optical coherence tomography (OCT) provides measurement of the thickness of the retinal nerve fiber layer (RNFL) and optic nerve head (ONH) parameters. RNFL thickness and ONH changes may reflect the changes that occur due to hypoxia in OSA. It was found that RNFL thinning was observed in OSA patients [20,21]. Theoretically, the severity of OSA will result in thinner RNFL and ONH parameter changes based on the severity of the hypoxia. However, there are also studies that have not been able to demonstrate the difference in the RNFL and ONH parameters changes in OSA $[22,23]$.

Our study aimed to compare the difference between mean RNFL thickness and ONH parameters in OSA patients and control. We also examined the potential relationship between RNFL thickness and ONH parameters and severity of OSA. We were also taking into consideration other factors that can also contribute to RNFL thinning; age, gender, obesity and smoking, which has not much been addressed in previous studies.

\section{Materials and Methods}

This observational cross-sectional study was conducted between May 2015 and February 2017. This was a prospective study. This study obtained ethical approval from the Human Research Ethics Committee of Universiti Sains Malaysia (USM/JEPeM/15050160) and was conducted in accordance with the Declaration of Helsinki for human research. All patients recruited has given their consent to participate in this study and for future publication of data.

\section{Patient selection}

Recruitment of OSA patients was conducted in the Department of Ophthalmology. A total of 54 OSA patients were recruited. Both newly diagnosed OSA and known cases on treatment with continuous positive airway pressure therapy (CPAP) were recruited. OSA was diagnosed based on apnoea-hypopnoea index (AHI) on polysomnogram. AHI of $\geq 5$ events $/ \mathrm{hr}$ was diagnosed of having sleep apnoea. The severity of OSA was divided into mild (AHI $5-15)$, moderate (16-30), and severe (>30). The control group consists of 54 individuals presented to Ophthalmology clinic without history of sleep apnoea and were selected based on simple random sampling method. Only OSA patients and control subject who have best corrected visual acuity of at least $6 / 12$ using the Snellen chart and clear ocular media with refractive error of not more than $+/-6.0$ diopters were included in this study. Subject who had a pre-existing optic neuropathy or optic disc hypoplasia, retinopathy, maculopathy, history of trauma or previous ocular surgery, and systemic disease of neurological and demyelinating diseases were excluded. All participants who consented to take part in the study undergo thorough ocular examinations and fundus evaluation via slit lamp biomicroscopy (Topcon, Tokyo, Japan). Intraocular pressure measurement was performed to rule out ocular pathology, which would have precluded participation in the study. Intraocular pressure was assessed with air-puff tonometer to ensure minimal manipulation to the cornea surface in order to prevent artifact on the OCT images. All participants were then subjected for OCT examinations for the right eye.

\section{OCT}

OCT examinations was performed using Cirrus OCT (Carl Zeiss Meditec, Dublin, CA, USA) using the fast RNFL scan protocol. The tests were performed by a single and well-trained operator. Only the test or repeated test that yielded signal strength of $\geq 6$ / 10 was taken for interpretations to ensure accuracy of the results. Measurement was taken on right eye which include average RNFL thickness, rim area, disc area, average cup/disc ratio, vertical cup/disc ratio, cup volume and RNFL thickness in four quadrant (superior, inferior, nasal, and temporal).

\section{Statistical analysis}

Data analysis was performed using the IBM SPSS Statistics ver. 22 (IBM Corp., Armonk, NY, USA). Descriptive analysis was used for the mean values and standard deviation (SD). All values will be tested for normal distribution in both groups. For demographic data, they will be tested for comparison of age, race and gender. The Student $t$-test, Pearson chi-square and Fisher exact test were used 
to analyse the demographic data. All $p$-values of $<0.05$ were considered statistically significant. Independent $t$-test were used to compare the means of $\mathrm{ONH}$ parameters and RNFL thickness between study group and control. A $p$-value of $<0.05$ was considered as significant. Pearson correlation were used to determine the correlations of ONH parameters and RNFL thickness and the AHI in the OSA group. The strength of association was determined and $p$-value of $<0.05$ was considered significant. General guidelines for assigning strength of association by Cohen will be used. Multivariate analysis of covariant (MANCOVA) analysis was done to perform regression analysis of systemic factors such as hypertension, diabetes, dyslipidemia, use of CPAP therapy and ocular factors of axial length and refractive error against the effect in RNFL thickness.

\section{Results}

There were a total of 108 participants. Among them 71 were male while 37 of them were female. The age of the participants ranged from 22 to 78 years. OSA patients have higher body mass index, more medical comorbidities and less number of active smoker compared to control subjects in Table 1. There were two OSA patients on CPAP therapy. The mean refractive error of both groups were equal at -0.04 . OSA patients has a slighter longer axial length at 23.01. Both groups had normal mean intraocular pressure at 15.3 for OSA patients and 14.7 for control patients. The mean RNFL values of OSA patients and controls are shown in Table 2. We observed a significant difference in the mean of average RNFL thickness with $p=0.008$ and superior thickness with $p \leq 0.001$ between the control and study group.

Table 1. Demographic data of OSA subjects and control

\begin{tabular}{|c|c|c|c|}
\hline & OSA $(n=54)$ & Control $(n=54)$ & $p$-value \\
\hline Mean age (yr) & $45.69 \pm 11.41$ & $42.54 \pm 13.13$ & $0.186^{*}$ \\
\hline \multicolumn{4}{|l|}{ Sex } \\
\hline Male & $35(32.4)$ & $36(33.3)$ & \multirow[t]{2}{*}{$0.839^{\dagger}$} \\
\hline Female & $19(17.6)$ & $18(16.7)$ & \\
\hline \multicolumn{4}{|l|}{ Race } \\
\hline Malay & $48(44.4)$ & $49(45.4)$ & \multirow[t]{2}{*}{$0.750^{\dagger}$} \\
\hline Chinese & $6(5.6)$ & $5(4.6)$ & \\
\hline Mean BMI & $33.04 \pm 6.29$ & $25.94 \pm 3.22$ & $<0.001^{*}$ \\
\hline \multicolumn{4}{|l|}{ Smoking status } \\
\hline Non-smoker & $50(46.3)$ & $37(34.3)$ & \multirow[t]{2}{*}{$0.003^{*}$} \\
\hline Smoker & $4(3.7)$ & $17(15.7)$ & \\
\hline \multicolumn{4}{|l|}{ Medical problem } \\
\hline Diabetes mellitus & $9(7.4)$ & $8(8.3)$ & $0.792^{\dagger}$ \\
\hline Hypertension & $19(17.8)$ & $11(10.3)$ & $0.075^{\dagger}$ \\
\hline Dyslipidemia & $18(16.8)$ & $10(9.3)$ & $0.069^{\dagger}$ \\
\hline Heart disease & $2(1.9)$ & $0(0.0)$ & $0.150^{\dagger}$ \\
\hline On CPAP therapy & $2(1.9)$ & $0(0.0)$ & $0.150^{\dagger}$ \\
\hline Refractive error (diopters) & $-0.04 \pm 0.19$ & $-0.04 \pm 0.19$ & $1.000^{*}$ \\
\hline Axial length & $23.01 \pm 0.54$ & $22.96 \pm 0.67$ & $0.670^{*}$ \\
\hline Intraocular pressure & $15.30 \pm 0.42$ & $14.70 \pm 0.39$ & $0.710^{*}$ \\
\hline
\end{tabular}

Values are presented as number or mean \pm standard deviation; The mean RNFL values of OSA patients and controls are shown in Table 2 ; We observed a significant difference in the mean of average RNFL thickness with $p=0.008$ and superior thickness with $p \leq 0.001$ between the control and study group.

OSA = obstructive sleep apnoea; BMI = body mass index; CPAP = continuous positive airway pressure therapy.

"Independent $t$-test; ${ }^{\dagger}$ Pearson chi-square test; ${ }^{*}$ Fisher exact test. 
Table 2. Comparing mean of RNFL thickness of right eye in OSA patient and control

\begin{tabular}{lcccccrc}
\hline $\begin{array}{l}\text { RNFL thickness } \\
(\mu \mathrm{m})\end{array}$ & $\begin{array}{c}\text { OSA } \\
(\mathrm{n}=54)\end{array}$ & $\begin{array}{c}\text { Control } \\
(\mathrm{n}=54)\end{array}$ & $\mathrm{t}$ & $\begin{array}{c}\text { Means } \\
\text { difference }\end{array}$ & $\begin{array}{c}95 \% \text { confidence } \\
\text { interval }\end{array}$ & $\mathrm{df}$ & $p$-value \\
\hline Average & $93.87 \pm 9.17$ & $98.96 \pm 10.50$ & 2.686 & 5.093 & $1.333-8.852$ & 104.12 & $0.008^{*}$ \\
Superior & $113.59 \pm 16.29$ & $125.76 \pm 14.93$ & 4.047 & 12.167 & $6.206-18.127$ & 105.20 & $<0.001^{*}$ \\
Inferior & $123.06 \pm 17.79$ & $126.80 \pm 16.02$ & 1.148 & 3.741 & $-2.719-10.200$ & 104.87 & 0.253 \\
Nasal & $69.65 \pm 9.61$ & $71.04 \pm 11.21$ & 0.691 & 1.389 & $-2.595-5.373$ & 103.59 & 0.490 \\
Temporal & $67.87 \pm 14.20$ & $72.09 \pm 12.78$ & 1.624 & 4.222 & $-0.932-9.376$ & 104.85 & 0.107 \\
\hline
\end{tabular}

Values are presented as mean \pm standard deviation unless otherwise indicated.

$\mathrm{RNFL}=$ retinal nerve fiber layer; OSA $=$ obstructive sleep apnoea.

*Independent $t$-test.

Table 3. Multivariate analysis of covariant of right eye RNFL thickness in obstructive sleep apnoea subject and control

\begin{tabular}{|c|c|c|c|c|c|c|}
\hline Effect & Value & $\mathrm{F}$ & Hypothesis df & Error df & Partial eta squared & Significance \\
\hline Age & 0.910 & 0.901 & 10.00 & 91.00 & 0.900 & 0.536 \\
\hline BMI & 0.950 & 0.477 & 10.00 & 91.00 & 0.500 & 0.901 \\
\hline Smoking & 0.841 & 1.722 & 10.00 & 91.00 & 0.159 & 0.088 \\
\hline Diabetes mellitus & 0.921 & 0.705 & 10.00 & 91.00 & 0.072 & 0.718 \\
\hline Hypertension & 0.901 & 0.996 & 10.00 & 91.00 & 0.099 & 0.453 \\
\hline Dyslipidemia & 0.827 & 1.900 & 10.00 & 91.00 & 0.173 & 0.055 \\
\hline CPAP therapy & 0.425 & 1.422 & 10.00 & 91.00 & 0.125 & 0.652 \\
\hline Refractive power & -0.021 & 0.377 & 10.00 & 91.00 & 0.004 & 0.426 \\
\hline Axial length & 0.850 & 0.822 & 10.00 & 91.00 & 0.500 & 0.567 \\
\hline
\end{tabular}

$\mathrm{RNFL}=$ retinal nerve fiber layer; $\mathrm{BMI}=$ body mass index; CPAP $=$ continuous positive airway pressure therapy.

Table 4. Comparing mean of ONH parameters of right eye in OSA patient and control

\begin{tabular}{llcccccc}
\hline ONH parameters & OSA $(\mathrm{n}=54)$ & Control $(\mathrm{n}=54)$ & $\mathrm{t}$ & $\begin{array}{c}\text { Means } \\
\text { difference }\end{array}$ & $\begin{array}{c}95 \% \text { confidence } \\
\text { interval }\end{array}$ & $\mathrm{df}$ & $p$-value \\
\hline RNFL symmetry $(\%)$ & $87.07 \pm 8.18$ & $83.67 \pm 9.92$ & 1.947 & 3.407 & $-6.879-0.640$ & 102.30 & 0.054 \\
Rim area & $1.303 \pm 0.214$ & $1.346 \pm 0.232$ & 1.002 & 0.043 & $-0.042-0.128$ & 105.34 & 0.319 \\
Disc area & $2.023 \pm 0.331$ & $2.123 \pm 0.421$ & 1.373 & 0.100 & $-0.044-0.244$ & 100.46 & 0.173 \\
Average CDR & $0.567 \pm 0.108$ & $0.563 \pm 0.163$ & 0.146 & 0.004 & $-0.567-0.049$ & 91.870 & 0.884 \\
Vertical CDR & $0.522 \pm 0.119$ & $0.524 \pm 0.162$ & 0.061 & 0.002 & $-0.053-0.056$ & 97.40 & 0.952 \\
Cup volume & $0.170 \pm 0.119$ & $0.214 \pm 0.155$ & 1.636 & 0.436 & $-0.009-0.096$ & 106.00 & 0.105 \\
\hline
\end{tabular}

Values are presented as mean \pm standard deviation unless otherwise indicated.

$\mathrm{ONH}=$ optic nerve head; OSA = obstructive sleep apnoea; RNFL = retinal nerve fiber layer; CDR $=$ cup to disc ratio.

*Independent $t$-test.

MANCOVA was performed to look into the possibilities of diabetes mellitus, hypertension, dyslipidemia and CPAP therapy becoming covariates affecting our results in Table 3. The MANCOVA analysis showed that the age, body mass index, smoking, diabetes mellitus, hypertension, dys- lipidemia and CPAP therapy were not the confounder affecting the thickness of RNFL in our study. For ocular factors such as axial length and refractive error, we found out that they were not confounding factors affecting RNFL thickness in our study. In our statistical analysis, all 
Table 5. Correlation between the RNFL thickness of right eye and apnoea-hypopnoea index in the obstructive sleep apnoea patients

\begin{tabular}{lccccc}
\hline & $\mathrm{R}$ & $\mathrm{R}^{2}$ & $\mathrm{~F}$ & $\mathrm{r}$ & $p$-value \\
\hline RNFL thickness & & & & & \\
Average & 0.228 & 0.052 & 2.845 & -0.228 & 0.980 \\
Superior & 0.295 & 0.087 & 4.962 & -0.295 & $0.030^{*}$ \\
Inferior & 0.043 & 0.002 & 0.097 & -0.043 & 0.756 \\
Nasal & 0.292 & 0.085 & 4.852 & -0.292 & $0.032^{*}$ \\
Temporal & 0.102 & 0.010 & 0.548 & -0.102 & 0.463 \\
ONH parameters & & & & & \\
Rim area & 0.029 & 0.001 & 0.043 & 0.029 & 0.836 \\
Disc area & 0.248 & 0.062 & 3.410 & 0.248 & 0.070 \\
Average CDR & 0.246 & 0.060 & 3.348 & 0.246 & 0.073 \\
Vertical CDR & 0.253 & 0.064 & 3.568 & 0.253 & 0.064 \\
Cup volume & 0.053 & 0.003 & 0.147 & 0.053 & 0.703 \\
\hline
\end{tabular}

$\mathrm{RNFL}=$ retinal nerve fiber layer; $\mathrm{ONH}=$ optic nerve head; $\mathrm{CDR}=$ cup to disc ratio.

*Simple linear regression and Pearson correlation $(p<0.05)$.

the independent variables were tested for multicollinearity and was found to be not significant.

Table 4 showed that there was no significant difference between mean of ONH parameters of OSA subjects and control.

Table 5 showed that there was a significantly fair negative correlation between AHI and RNFL thickness among the subject in the OSA group in the superior $\mathrm{R}=0.295, \mathrm{R}^{2}$ $=0.087, \mathrm{r}=-0.295, p=0.030(p<0.05)$ and nasal quadrant $\mathrm{R}=0.292, \mathrm{R}^{2}=0.085, \mathrm{r}=-0.292, p=0.032(p<0.05)$. However, none of the ONH parameters had significant linear relationship with the AHI among subjects in the OSA group.

\section{Discussion}

OSA is a sleep-related condition which causes a decrease in the arterial oxygen saturation and a rise in the carbon dioxide saturation during sleep. Many authors hypothesized that chronic oxygen deprivation is responsible for the underlying pathophysiology of the disease, resulting in generalized sympathetic activation and metabolic dysregulation, subsequently leading to the increase risk of cardiovascular and cerebrovascular diseases [24-26]. In ocular tissues, the same pathophysiology occurs, causing a reduction of ocular perfusion pressure and decreased and ischaemia to the ganglion cell layer, leading to optic neuropathy.

Our result showed a significant difference in the average RNFL thickness with a mean difference of 5.093, $p=0.008$ $(<0.05)$ and superior thickness with a mean difference of 12.167, $p<0.001(p<0.05)$ between the control and study group. This is similar to the results of Sagiv et al. [21] who found significant thinning of RNFL in all quadrants except nasally in moderate to severe OSA subjects and Lin et al. [27] found significant thinning in the superior quadrant. Likewise, similar findings were also reported by Kargi et al. [28]. Our findings are in contrast to the findings of Adam et al. [22] and Nowak et al. [23], who found no significant changes in RNFL thickness in OSA. Kargi et al. [28] demonstrated that decreased ocular perfusion secondary to hypoxia and vasospasm may lead to RNFL thinning. Although many researchers found a relatively thinner RNFL in OSA subjects, the quadrant involved were not similar. We think that this is due to the possibility that some sub-clinical papilledema may actually happen in the early part of the disease [29]. When the oedema of the ganglion cell layer resolved, the resolution of the oedema may not be symmetrical or occurred at the same time in all quadrant, hence asymmetrical thinning of the quadrant RNFL happen.

We went a step further by performing a multivariate 
analysis of covariant to determine whether our significant result was also contributed by any confounding factor. It has been shown in previous study that RNFL thickness was reduced in diabetes mellitus, hypertension, dyslipidemia, increased body mass index, age, smoking habit and CPAP therapy [30-32]. In our study, any of the above factors were not found to be a confounder. For ocular parameters, such as refractive error and axial length, our study analysis showed that they were not confounding factors as well.

We found no significant difference with regards to $\mathrm{ONH}$ parameters between OSA and control. This is similar to the study by Lin et al, who found no significant differences of ONH parameters between OSA and controls. Contrary to the findings by Casas et al. [20], they found larger disc area in her OSA group of patients while Huseyinoglu et al. [31] found that nerve head volume in his mild and moderate OSA group had higher reading compared to control group and decrease in severe group compare to control. They postulated that this probably happen due to optic disc had already pass-through stage of subtle oedema in the mild and moderate group and move into the stage of reduction of RNFL thickness with axonal loss in severe group. We have almost half of our sample in the severe group, therefore the supposedly increase volume in the mild and moderate group had been compensated by the reduction in the thickness in our severe group, hence probably normalising or reducing our mean difference.

Our regression analysis showed a fair significant negative direct linear relationship between the RNFL thickness in the superior and nasal quadrant with the AHI. For every increment of $1 \mathrm{AHI}$ index, the decrement of 0.295 micron occur in the superior quadrant and 0.292 micron in the nasal quadrant. These results are similar to studies by Lin et al. [27], Moghimi et al. [33], and Sagiv et al. [21] who also found correlation between the nasal quadrant and severity, but they used respiratory disturbance index instead of AHI [34]. However, in our study, there are no significant correlations and associations between any of the $\mathrm{ONH}$ parameters and AHI.

We concluded that abnormalities in $\mathrm{ONH}$ parameters occur secondary to RNFL loss; thus, RNFL changes may be a more sensitive marker of hypoxia in OSA compared to the optic changes of nerve head parameters.

Due to limited number of patient available to participate in our study, this study also include patients who were newly diagnosed as well as those who were already on treatment with CPAP. Therefore, the result may not be as accurate as taking only a newly diagnosed OSA, as the RNFL thickness had also been shown to be decreasing after treatment with CPAP therapy [33]. It was postulated to occur secondary to CPAP therapy causes increased in intraocular pressure at night $[35,36]$, therefore, these group of patients may have higher risk of developing glaucoma. In future, this limitation may be overcome by taking only the newly diagnosed OSA before the initiation of any treatment.

We are unable to determine the exact duration of the OSA. However, majority of our subjects were diagnosed to have OSA within 2 years of diagnosis when we performed OCT examination on them. It is possible that the greater the severity and longer duration of the hypoxia, the greater the RNFL alterations. Furthermore, a referral bias could be present since patients were referred for snoring and, therefore, they might not be representative of the entire OSA population which might be mostly undiagnosed. It is possible that the greater the severity with longer duration of the hypoxia will result in greater the RNFL alterations. Our sample size of 54 patients was not enough to represent OSA population as a whole and to overcome the short duration of diagnosis of the patients. In future cohort, we planned to extend the study to 5 years to include patients with longer duration of OSA, therefore enabling us to include the duration as part of the analysis.

Our study is a cross-sectional, prospective study. There were no comparison done or follow up to actually look at the changes in all of the studied parameters over time. We suggest that in future, another study to be done on these patients in order to evaluate the possible changes on the RNFL thickness as well as ONH parameters in these patients.

Our study is limited by the presence of medical problem in our control. We were unable to fully exclude those with medical problem from becoming our control group. Therefore, the result may be confounded by the presence of these factors. We suggest that if similar study will be carried out in the future should, the control group should be selected among those without medical problem.

Our study showed that OSA patients have reduced RNFL thickness in the average and superior quadrant compared to controls. A significant fair direct negative correlation exists between superior quadrant as well as na- 
sal quadrant of RNFL with the severity of the disease, measured by the AHI. Further evaluations and long term follow up may be needed in future in order to monitor the progression of RNFL thinning in this group of patients.

\section{Conflict of Interest}

No potential conflict of interest relevant to this article was reported.

\section{Acknowledgements}

We would like to extend out largest gratitude and our sincere appreciation to the otorhinolaryngology and ophthalmology staffs in HUSM for their assistance in this study.

\section{References}

1. American Academy of Sleep Medicine. International classification of sleep disorders: diagnostic and coding manual. 2nd ed. Westchester: American Academy of Sleep Medicine; 2005. p. 211-14.

2. Puvanendran K, Goh KL. From snoring to sleep apnea in a Singapore population. Sleep Res Online 1999;2:11-4.

3. Asaoka S, Namba K, Tsuiki S, et al. Excessive daytime sleepiness among Japanese public transportation drivers engaged in shiftwork. J Occup Environ Med 2010;52:813-8.

4. Ip MS, Lam B, Lauder IJ, et al. A community study of sleep-disordered breathing in middle-aged Chinese men in Hong Kong. Chest 2001;119:62-9.

5. Ip MS, Lam B, Tang LC, et al. A community study of sleep-disordered breathing in middle-aged Chinese women in Hong Kong: prevalence and gender differences. Chest 2004;125:127-34.

6. Arikawa T, Toyoda S, Haruyama A, et al. Impact of obstructive sleep apnoea on heart failure with preserved ejection fraction. Heart Lung Circ 2016;25:435-41.

7. Kent BD, McNicholas WT, Ryan S. Insulin resistance, glucose intolerance and diabetes mellitus in obstructive sleep apnoea. J Thorac Dis 2015;7:1343-57.

8. Shastri A, Bangar S, Holmes J. Obstructive sleep apnoea and dementia: is there a link? Int J Geriatr Psychiatry 2016;31:400-5.

9. Mojon DS, Hedges TR 3rd, Ehrenberg B, et al. Association between sleep apnea syndrome and nonarteritic anterior ischemic optic neuropathy. Arch Ophthalmol 2002;120:6015.

10. Palombi K, Renard E, Levy P, et al. Non-arteritic anterior ischaemic optic neuropathy is nearly systematically associated with obstructive sleep apnoea. $\mathrm{Br} J$ Ophthalmol 2006;90:879-82.

11. Bilgin G, Koban Y, Arnold AC. Nonarteritic anterior ischemic optic neuropathy and obstructive sleep apnea. $J \mathrm{Neu}$ roophthalmol 2013;33:232-4.

12. Bendel RE, Kaplan J, Heckman M, et al. Prevalence of glaucoma in patients with obstructive sleep apnoea: a cross-sectional case-series. Eye (Lond) 2008;22:1105-9.

13. Tsang CS, Chong SL, Ho CK, Li MF. Moderate to severe obstructive sleep apnoea patients is associated with a higher incidence of visual field defect. Eye (Lond) 2006;20:3842.

14. Faridi O, Park SC, Liebmann JM, Ritch R. Glaucoma and obstructive sleep apnoea syndrome. Clin Exp Ophthalmol 2012;40:408-19.

15. Mojon DS, Hess CW, Goldblum D, et al. Primary open-angle glaucoma is associated with sleep apnea syndrome. Ophthalmologica 2000;214:115-8.

16. Glacet-Bernard A, Leroux les Jardins G, Lasry S, et al. Obstructive sleep apnea among patients with retinal vein occlusion. Arch Ophthalmol 2010;128:1533-8.

17. Leroux les Jardins G, Glacet-Bernard A, Lasry S, et al. Retinal vein occlusion and obstructive sleep apnea syndrome. J Fr Ophtalmol 2009;32:420-4.

18. Kloos P, Laube I, Thoelen A. Obstructive sleep apnea in patients with central serous chorioretinopathy. Graefes Arch Clin Exp Ophthalmol 2008;246:1225-8.

19. Fraser CL. Obstructive sleep apnea and optic neuropathy: is there a link? Curr Neurol Neurosci Rep 2014;14:465.

20. Casas P, Ascaso FJ, Vicente E, et al. Retinal and optic nerve evaluation by optical coherence tomography in adults with obstructive sleep apnea-hypopnea syndrome (OSAHS). Graefes Arch Clin Exp Ophthalmol 2013;251: 1625-34.

21. Sagiv O, Fishelson-Arev T, Buckman G, et al. Retinal nerve fibre layer thickness measurements by optical coherence tomography in patients with sleep apnoea syndrome. Clin Exp Ophthalmol 2014;42:132-8. 
22. Adam M, Okka M, Yosunkaya S, et al. The evaluation of retinal nerve fiber layer thickness in patients with obstructive sleep apnea syndrome. J Ophthalmol 2013;2013:292158.

23. Nowak MS, Jurowski P, Gos R, et al. Pulsatile ocular blood flow in subjects with sleep apnoea syndrome. Arch Med Sci 2011;7:332-6.

24. Fletcher EC. Cardiovascular disease associated with obstructive sleep apnea. Monaldi Arch Chest Dis 2003;59:25461.

25. Fletcher EC. Sympathetic over activity in the etiology of hypertension of obstructive sleep apnea. Sleep 2003;26:159.

26. Jean-Louis G, Zizi F, Clark LT, et al. Obstructive sleep apnea and cardiovascular disease: role of the metabolic syndrome and its components. J Clin Sleep Med 2008;4:26172.

27. Lin PW, Friedman M, Lin HC, et al. Decreased retinal nerve fiber layer thickness in patients with obstructive sleep apnea/hypopnea syndrome. Graefes Arch Clin Exp Ophthalmol 2011;249:585-93.

28. Kargi SH, Altin R, Koksal M, et al. Retinal nerve fibre layer measurements are reduced in patients with obstructive sleep apnoea syndrome. Eye (Lond) 2005;19:575-9.

29. Purvin VA, Kawasaki A, Yee RD. Papilledema and obstructive sleep apnea syndrome. Arch Ophthalmol 2000;118:1626-30.

30. Kardys A, Weinstock-Guttman B, Dillon M, et al. Choles- terol affects retinal nerve fiber layer thickness in patients with multiple sclerosis with optic neuritis. Eur J Neurol 2013;20:1264-71.

31. Huseyinoglu N, Ekinci M, Ozben S, et al. Optic disc and retinal nerve fiber layer parameters as indicators of neurodegenerative brain changes in patients with obstructive sleep apnea syndrome. Sleep Breath 2014;18:95-102.

32. Dogan B, Kazim Erol M, Dogan U, et al. The retinal nerve fiber layer, choroidal thickness, and central macular thickness in morbid obesity: an evaluation using spectral-domain optical coherence tomography. Eur Rev Med Pharmacol Sci 2016;20:886-91.

33. Moghimi S, Ahmadraji A, Sotoodeh H, et al. Retinal nerve fiber thickness is reduced in sleep apnea syndrome. Sleep Med 2013;14:53-7.

34. Moschos MM, Nitoda E, Laios K, et al. The impact of chronic tobacco smoking on retinal and choroidal thickness in greek population. Oxid Med Cell Longev 2016;2016: 2905789.

35. Zengin MO, Tuncer I, Karahan E. Retinal nerve fiber layer thickness changes in obstructive sleep apnea syndrome: one year follow-up results. Int J Ophthalmol 2014;7:704-8.

36. Kiekens S, Veva De Groot, Coeckelbergh T, et al. Continuous positive airway pressure therapy is associated with an increase in intraocular pressure in obstructive sleep apnea. Invest Ophthalmol Vis Sci 2008;49:934-40. 